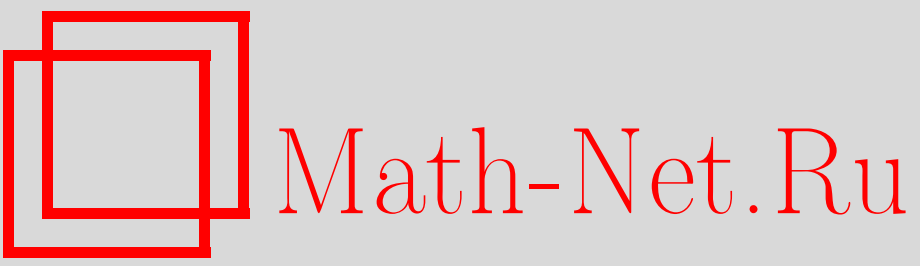

М. Ю. Балахнев, Об одном классе интегрируемых эволюционных векторных уравнений, ТМФ, 2005, том 142, номер 1, 13-20

DOI: https://doi.org/10.4213/tmf1759

Использование Общероссийского математического портала Math-Net.Ru подразумевает, что вы прочитали и согласны с пользовательским соглашением

http://www.mathnet.ru/rus/agreement

Параметры загрузки:

IP : 35.173 .137 .237

26 апреля 2023 г., 10:52:52 
ТЕОРЕТИЧЕСКАЯ

И МАТЕМАТИЧЕСКАЯ

ФИЗИКА

Том 142, № 1

январь, 2005

(C) 2005 г.

М. Ю. Балахнев*

\section{ОБ ОДНОМ КЛАССЕ ИНТЕГРИРУЕМЫХ ЭВОЛЮЦИОННЫХ ВЕКТОРНЫХ УРАВНЕНИЙ}

Представлены результаты классификации интегрируемых эволюционных $N$-компонентных векторных уравнений. В качестве доказательства точной интегрируемости построены преобразования Беклунда для каждого уравнения.

Ключевые слова: векторные эволюционные уравнения, преобразования Беклунда.

\section{1. ВВЕДЕНИЕ}

Первые примеры интегрируемых векторных уравнений как векторно-матричных обобщений известных уравнений и систем были опубликованы в работе [1]. Среди них широко известны два векторных обобшения модифицированного уравнения Кортевега-де Фриза (мКдФ):

$$
\begin{aligned}
& \mathbf{u}_{t}=\mathbf{u}_{x x x}+(\mathbf{u}, \mathbf{u}) \mathbf{u}_{x} \\
& \mathbf{u}_{t}=\mathbf{u}_{x x x}+(\mathbf{u}, \mathbf{u}) \mathbf{u}_{x}+\left(\mathbf{u}, \mathbf{u}_{x}\right) \mathbf{u}
\end{aligned}
$$

где $\mathbf{u}(t, x)$ есть вектор $N$-мерного векторного пространства $V$ со скалярным произведением $(\cdot, \cdot)$. Известным примером яляется также векторное обобшение высшей симметрии уравнения Ландау-Лифшица [2]:

$$
\mathbf{u}_{t}=\left(\mathbf{u}_{x x}+\frac{3}{2}\left(\mathbf{u}_{x}, \mathbf{u}_{x}\right) \mathbf{u}\right)_{x}+\frac{3}{2}\langle\mathbf{u}, \mathbf{u}\rangle \mathbf{u}_{x}, \quad \mathbf{u}^{2}=1
$$

Здесь и далее по тексту $\langle\cdot, \cdot\rangle$ - второе скалярное произведение, задаваемое формулой $\langle\mathbf{u}, \mathbf{v}\rangle=(\mathbf{u}, R \mathbf{v})$, где $R$ - постоянная симметричная матрица.

Классификация векторных полиномиальных уравнений была проведена в работе [3]. В статье [4] были разработаны общие принципы симметрийного подхода в классификации векторных уравнений и проведена классификация уравнений на $n$-мерной сфере. В работе [5] получены векторные уравнения, имеюшие вид законов сохранения.

\footnotetext{
* Орловский государственный университет, Орел, Россия. E-mail: maxibal@yandex.ru
} 
Мы приводим результаты классификации уравнений вида

$$
\begin{aligned}
\mathbf{u}_{t} & =\mathbf{u}_{x x x}+f_{1} \mathbf{u}_{x}+f_{0} \mathbf{u}, & & f_{k}=f_{k}\left(u_{[i, j]}\right), \\
u_{[i, j]} & =\left(\frac{\partial^{i} \mathbf{u}}{\partial x^{i}}, \frac{\partial^{j} \mathbf{u}}{\partial x^{j}}\right), & & 0 \leqslant i \leqslant j \leqslant 2 .
\end{aligned}
$$

\section{2. РЕЗУЛЬТАТЫ КЛАССИФИКАЦИИ}

Согласно рекуррентной формуле для канонических сохраняющихся плотностей $\rho_{k}[4]$, для системы (3) можно легко установить, что функция $f_{0}$ должна иметь вид полной производной $f_{0} \in \operatorname{Im} D$. Дальнейший анализ условий интегрируемости мы провели с помощью Maple-программ [6] и проверили шесть условий интегрируемости $D_{t} \rho_{k} \in \operatorname{Im} D$, $k=0, \ldots, 5$. Произведенные на компьютере промежуточные вычисления опустим ввиду их громоздкости и приведем только конечный результат.

ТЕОрема 1. Если уравнение (3) допускает бесконечную последовательность законов сохранения $\left(\rho_{k}\right)_{t}=\left(\sigma_{k}\right)_{x}, k \rightarrow \infty$, где $\rho_{k}$ и $\sigma_{k}-$ функции переменных $u_{[i, j]}$, то әто уравнение принадлежит следующему списку:

$$
\begin{aligned}
& \mathbf{u}_{t}=\mathbf{u}_{x x x}+u_{[0,0]} \mathbf{u}_{x} ; \\
& \mathbf{u}_{t}=\mathbf{u}_{x x x}+u_{[0,0]} \mathbf{u}_{x}+u_{[0,1]} \mathbf{u} \text {; } \\
& \mathbf{u}_{t}=\mathbf{u}_{x x x}+\frac{3}{2}\left(\left(\frac{u_{[1,1]}}{a}+\frac{u_{[0,1]}^{2}}{a\left(a-u_{[0,0]}\right)}\right) \mathbf{u}\right)_{x}+\frac{3}{2} b u_{[0,0]} \mathbf{u}_{x} ; \\
& \mathbf{u}_{t}=\mathbf{u}_{x x x}+\frac{3}{2}\left(\left(\frac{u_{[1,1]}}{a}+\frac{u_{[0,1]}^{2}}{a\left(a-u_{[0,0]}\right)}\right) \mathbf{u}\right)_{x}+\frac{3}{2}\left(\frac{u_{[1,1]}}{a}+\frac{u_{[0,1]}^{2}}{a\left(a-u_{[0,0]}\right)}\right) \mathbf{u}_{x} \text {; } \\
& \mathbf{u}_{t}=\mathbf{u}_{x x x}+\frac{3}{2}\left(\left(\frac{u_{[1,1]}}{a}+\frac{u_{[0,1]}^{2}}{a\left(a-u_{[0,0]}\right)}\right) \mathbf{u}\right)_{x}+\frac{3 u_{[0,2]}}{a-u_{[0,0]}} \mathbf{u}_{x}+ \\
& +\frac{3}{a-u_{[0,0]}}\left(\frac{u_{[1,1]} u_{[0,0]}}{a}+\frac{u_{[0,1]}^{2}\left(a+2 u_{[0,0]}\right)}{2 a\left(a-u_{[0,0]}\right)}-b u_{[0,0]}\right) \mathbf{u}_{x} \\
& \mathbf{u}_{t}=\mathbf{u}_{x x x}+\frac{3}{2}\left(\left(\frac{u_{[1,1]}}{a}+\frac{u_{[0,1]}^{2}+b}{a\left(a-u_{[0,0]}\right)}\right) \mathbf{u}\right)_{x}+\frac{3 u_{[0,2]}}{a-u_{[0,0]}} \mathbf{u}_{x}- \\
& -\frac{3}{2} \frac{a-3 u_{[0,0]}}{a-u_{[0,0]}}\left(\frac{u_{[1,1]}}{a}+\frac{u_{[0,1]}^{2}+b}{a\left(a-u_{[0,0]}\right)}\right) \mathbf{u}_{x} \text {; } \\
& \mathbf{u}_{t}=\mathbf{u}_{x x x}-\frac{3 a}{2}\left(u_{[2,2]}-\frac{a\left(1+b u_{[0,0]}\right) u_{[1,2]}^{2}}{\zeta}-\right. \\
& \left.-\frac{2 b u_{[0,1]} u_{[1,2]}\left(1-a u_{[0,2]}\right)}{\zeta}-\frac{b\left(1-a u_{[0,2]}\right)^{2}\left(\zeta+a b u_{[0,1]}^{2}\right)}{a^{2}\left(1+b u_{[0,0]}\right) \zeta}\right) \mathbf{u}_{x} \\
& \mathbf{u}_{t}=\mathbf{u}_{x x x}-\frac{3 a}{2}\left(u_{[2,2]}-\frac{a u_{[0,0]} u_{[1,2]}^{2}}{\xi}-\right.
\end{aligned}
$$


ОБ ОДНОМ КЛАССЕ ИНТЕГРИРУЕМЫХ ЭВОЛЮЦИОННЫХ ВЕКТОРНЫХ УРАВНЕНИЙ 15

$$
\begin{aligned}
& \left.-\frac{2 u_{[0,1]}\left(1-a u_{[0,2]}\right) u_{[1,2]}}{\xi}-\frac{\left(1-a u_{[0,2]}\right)^{2}\left(\xi+a u_{[0,1]}^{2}\right)}{a^{2} u_{[0,0]} \xi}\right) \mathbf{u}_{x} \\
& \mathbf{u}_{t}=\mathbf{u}_{x x x}+\frac{3}{2}\left(u_{[1,1]}-\frac{u_{[0,1]}^{2}}{1+u_{[0,0]}}\right) \mathbf{u}_{x}-\frac{3}{2}\left(u_{[1,1]}-\frac{u_{[0,1]}^{2}}{1+u_{[0,0]}}\right)_{x} \mathbf{u}- \\
& -\frac{3 a}{2}\left(u_{[2,2]}-\frac{a u_{[0,0]} u_{[1,2]}^{2}}{\xi}-\frac{2 u_{[0,1]}\left(1-a u_{[0,2]}\right) u_{[1,2]}}{\xi}-\right. \\
& \left.-\frac{\left(1-a u_{[0,2]}\right)^{2}\left(\xi+a u_{[0,1]}^{2}\right)}{a^{2} u_{[0,0]} \xi}\right) \mathbf{u}_{x} \text {; } \\
& \mathbf{u}_{t}=\mathbf{u}_{x x x}+3\left(\frac{\sqrt{\xi} u_{[0,1]}}{\sqrt{-a} u_{[0,0]}^{2}}-\frac{\xi-a u_{[0,1]}^{2}}{2 a u_{[0,0]}^{2}}\right) \mathbf{u}_{x}-3\left(\frac{\sqrt{\xi} u_{[0,1]}}{\sqrt{-a} u_{[0,0]}^{2}}-\frac{\xi-a u_{[0,1]}^{2}}{2 a u_{[0,0]}^{2}}\right)_{x} \mathbf{u}- \\
& -\frac{3 a}{2}\left(u_{[2,2]}-\frac{a u_{[0,0]} u_{[1,2]}^{2}}{\xi}-\frac{2 u_{[0,1]}\left(1-a u_{[0,2]}\right) u_{[1,2]}}{\xi}-\right. \\
& \left.-\frac{\left(1-a u_{[0,2]}\right)^{2}\left(\xi+a u_{[0,1]}^{2}\right)}{a^{2} u_{[0,0]} \xi}\right) \mathbf{u}_{x}
\end{aligned}
$$

где а и $b$ - произвольные константы, $\zeta=\left(1+a u_{[1,1]}\right)\left(1+b u_{[0,0]}\right)-a b u_{[0,1]}^{2}, \quad \xi=$ $u_{[0,0]}\left(1+a u_{[1,1]}\right)-a u_{[0,1]}^{2}$.

Очевидно, что система (3) инвариантна относительно движений пространства $V$, поэтому, как и в работах [4], [5], назовем такие системы изотропными. Расширим множество динамических переменных $u_{[i, j]}$ путем добавления анизотропных переменных

$$
\tilde{u}_{[i, j]}=\left\langle\frac{\partial^{i} \mathbf{u}}{\partial x^{i}}, \frac{\partial^{j} \mathbf{u}}{\partial x^{j}}\right\rangle, \quad 0 \leqslant i \leqslant j \leqslant n .
$$

Теорема 2. Если уравнение (3) удовлетворяет условию теоремъ 1 с функииями $\rho_{i}$ и $\sigma_{i}$, зависячими от $u_{[i, j]}$ и $\tilde{u}_{[i, j]}$, а коэффициенты $f_{1}$ и $f_{0}$ содержат переменные (14) при $n=1$, то это уравнение принадлежит следующему списку:

$$
\begin{aligned}
\mathbf{u}_{t}= & \mathbf{u}_{x x x}+\frac{3}{2}\left(\left(\frac{u_{[1,1]}}{a}+\frac{u_{[0,1]}^{2}}{a\left(a-u_{[0,0]}\right)}\right) \mathbf{u}\right)_{x}+\frac{3}{2} \tilde{u}_{[0,0]} \mathbf{u}_{x} ; \\
\mathbf{u}_{t}= & \mathbf{u}_{x x x}+\frac{3}{2}\left(\tilde{u}_{[1,1]}-\frac{\tilde{u}_{[0,1]}^{2}}{1+\tilde{u}_{[0,0]}}\right) \mathbf{u}_{x}-\frac{3}{2}\left(\tilde{u}_{[1,1]}-\frac{\tilde{u}_{[0,1]}^{2}}{1+\tilde{u}_{[0,0]}}\right)_{x} \mathbf{u}- \\
& -\frac{3 a}{2}\left(u_{[2,2]}-\frac{a u_{[0,0]} u_{[1,2]}^{2}}{\xi}-\frac{2 u_{[0,1]}\left(1-a u_{[0,2]}\right) u_{[1,2]}}{\xi}-\right. \\
& \left.-\frac{\left(1-a u_{[0,2]}\right)^{2}\left(\xi+a u_{[0,1]}^{2}\right)}{a^{2} u_{[0,0]} \xi}\right) \mathbf{u}_{x} \\
\mathbf{u}_{t}= & \mathbf{u}_{x x x}+3 h \mathbf{u}_{x}-3 h_{x} \mathbf{u}-
\end{aligned}
$$




$$
\begin{aligned}
& -\frac{3 a}{2}\left(u_{[2,2]}-\frac{a u_{[0,0]} u_{[1,2]}^{2}}{\xi}-\frac{2 u_{[0,1]}\left(1-a u_{[0,2]}\right) u_{[1,2]}}{\xi}-\right. \\
& \left.-\frac{\left(1-a u_{[0,2]}\right)^{2}\left(\xi+a u_{[0,1]}^{2}\right)}{a^{2} u_{[0,0]} \xi}\right) \mathbf{u}_{x},
\end{aligned}
$$

где $a= \pm 1, \quad \xi=u_{[0,0]}\left(1+a u_{[1,1]}\right)-a u_{[0,1]}^{2}$,

$$
\begin{gathered}
h=\tilde{u}_{[1,1]}-2 \frac{u_{[0,1]} \tilde{u}_{[0,1]}}{u_{[0,0]}}+\sqrt{\xi} \frac{u_{[0,1]}-2\left(\tilde{u}_{[0,1]} u_{[0,0]}-u_{[0,1]} \tilde{u}_{[0,0]}\right)}{\sqrt{-a} u_{[0,0]}^{2}}- \\
-\frac{\left(1+2 \tilde{u}_{[0,0]}\right)\left(\xi-a u_{[0,1]}^{2}\right)}{2 a u_{[0,0]}^{2}}
\end{gathered}
$$

ЗАмечАниЕ. В уравнениях (6)-(13) константа $a$ не равна нулю и приводится к $a=$ \pm 1 подходяшим масштабным преобразованием $\mathbf{u}$. Постоянная $b$ в уравнениях (6), (8) и (9) либо равна нулю, либо приводится к $b=1$ растяжением по осям $t$ и $x$. Уравнения (6) и (10) в случае $b=0$ уже были получены в работе [5]. Уравнение (11) есть предельный случай уравнения (10) при фиксированном $a$ и $b \rightarrow \infty$.

\section{3. ДИФФЕРЕНЦИАЛЬНЫЕ ПОДСТАНОВКИ И ПРЕОБРАЗОВАНИЯ БЕКЛУНДА}

Мы установили, что системы (4), (5) связаны дифференциальными подстановками с системами (8), (9), соответственно. Уравнения (4) и (8) связаны подстановкой

$$
\mathbf{u}=\sqrt{-\frac{3}{2 a}}\left(\mathbf{v}_{x}+\frac{v_{[0,1]}-\sqrt{2 a b\left(a-v_{[0,0]}\right)}}{a-v_{[0,0]}} \mathbf{v}\right),
$$

где $\mathbf{u}$ удовлетворяет уравнению (4), а $\mathbf{v}$ - уравнению (8). Подстановка

$$
\mathbf{u}=\sqrt{-\frac{3}{a}}\left(\mathbf{v}_{x}+\frac{v_{[0,1]}+\sqrt{b}}{a-v_{[0,0]}} \mathbf{v}\right)
$$

связывает решение $\mathbf{u}$ уравнения (5) с решением $\mathbf{v}$ уравнения (9).

Алгоритм построения преобразований Беклунда описан в работах [4], [5]. Напомним только, что преобразования Беклунда для векторных уравнений зависят еше и от переменных

$$
w_{i}=\left(\mathbf{u}, \frac{\partial^{i} \mathbf{v}}{\partial x^{i}}\right), \quad \widetilde{w}_{i}=\left\langle\mathbf{u}, \frac{\partial^{i} \mathbf{v}}{\partial x^{i}}\right\rangle, \quad 0 \leqslant i \leqslant 1 .
$$

Здесь и далее $\mathbf{u}, \mathbf{v}$ - два решения одного уравнения, $\mu$ - параметр.

Первое векторное уравнение мКд $\Phi$ (4) имеет преобразование Беклунда вида

$$
\sqrt{6}\left(\mathbf{u}_{x}+\mathbf{v}_{x}\right)=(\mathbf{u}-\mathbf{v}) \sqrt{\mu-\left(u_{[0,0]}+v_{[0,0]}+2 w_{0}\right)},
$$




$$
\begin{aligned}
\sqrt{3}\left(\mathbf{u}_{x}+\mathbf{v}_{x}\right) & =\mu(\mathbf{u}-\mathbf{v})- \\
- & \frac{\mu+\sqrt{\mu^{2}-\left(u_{[0,0]}+v_{[0,0]}+2 w_{0}\right)}}{u_{[0,0]}+v_{[0,0]}+2 w_{0}} \\
& \left(\mathbf{u}\left(u_{[0,0]}+w_{0}\right)-\mathbf{v}\left(v_{[0,0]}+w_{0}\right)\right) .
\end{aligned}
$$

Преобразования (18) и (19) в скалярном пределе переходят в известное преобразование Беклунда для мКдФ

$$
\frac{u_{x}+v_{x}}{u-v}=k \sqrt{\mu-(u+v)^{2}} .
$$

Для уравнения (7) преобразование Беклунда записывается следующим образом:

$\mathbf{u}_{x}+\mathbf{v}_{x}=\frac{q w_{1}+p v_{[0,1]}}{q\left(a+p q+w_{0}\right)}(\mathbf{u}+\mathbf{v})+\mu\left(a \mathbf{v}-\left(p q+w_{0}\right) \mathbf{u}\right), \quad p^{2}=u_{[0,0]}-a, q^{2}=v_{[0,0]}-a$.

Преобразование Беклунда для уравнения (10) имеет вид

$$
\mathbf{u}_{x}-\mathbf{v}_{x}=\left(\frac{f \eta}{1+b v_{[0,0]}}-\frac{b\left(1+\mu w_{0}\right)-\mu}{1+b v_{[0,0]}} v_{[0,1]}+\mu w_{1}\right)(\mathbf{u}+\mathbf{v})
$$

где $f^{2}=b \mu^{2}\left(u_{[0,0]} v_{[0,0]}-w_{0}^{2}\right)-b\left(1+2 \mu w_{0}\right)+2 \mu+\mu^{2}\left(u_{[0,0]}+v_{[0,0]}+2 w_{0}\right), a \eta^{2}=(1+$ $\left.a v_{[1,1]}\right)\left(1+b v_{[0,0]}\right)-a b v_{[0,1]}^{2}$.

Предельный переход в уравнении $(21)$ при $b \rightarrow \infty$ позволяет, согласно замечанию, получить преобразование Беклунда для уравнения (11):

$$
\mathbf{u}_{x}-\mathbf{v}_{x}=\left(\frac{f \eta}{v_{[0,0]}}-\frac{1+\mu w_{0}}{v_{[0,0]}} v_{[0,1]}+\mu w_{1}\right)(\mathbf{u}+\mathbf{v}),
$$

где $f^{2}=\mu^{2}\left(v_{[0,0]} u_{[0,0]}-w_{0}^{2}\right)-\left(1+2 \mu w_{0}\right), a \eta^{2}=v_{[0,0]}\left(1+a v_{[1,1]}\right)-a v_{[0,1]}^{2}$.

Можно заметить, что при равенстве скалярных произведений $\langle\cdot, \cdot\rangle=(\cdot, \cdot)$ уравнения (6) и (15), (12) и (16), (13) и (17) совпадают. Следовательно, преобразования Беклунда для уравнений $(6),(12)$ и (13) можно получить из преобразований Беклунда для $(15),(16)$ и $(17)$, соответственно, положив $\tilde{u}_{[i, j]}=u_{[i, j]}$ и $\tilde{w}_{i}=w_{i}$. В связи с этим мы приводим преобразования Беклунда только для анизотропных систем (15)-(17).

Для анизотропного уравнения (15) преобразование Беклунда записывается в виде

$$
\begin{aligned}
\mathbf{u}_{x}+\mathbf{v}_{x}= & \frac{q w_{1}+p v_{[0,1]}}{q\left(a+p q+w_{0}\right)}(\mathbf{u}+\mathbf{v})+ \\
& +\frac{\sqrt{\mu\left(a+p q+w_{0}\right)-\tilde{u}_{[0,0]}-\tilde{v}_{[0,0]}-2 \widetilde{w}_{0}}}{a+p q+w_{0}}\left(a \mathbf{v}-\left(p q+w_{0}\right) \mathbf{u}\right),
\end{aligned}
$$

где $p$ и $q$ те же, что и в $(20)$. 
Преобразование Беклунда для уравнения (16) имеет вид

$$
\begin{aligned}
\mathbf{u}_{x}-\mathbf{v}_{x}= & \left(\widetilde{w}_{1}+\frac{\tilde{p}}{\tilde{q}} \tilde{v}_{[0,1]}\right) \mathbf{u}+ \\
& +\left(\frac{f \eta}{v_{[0,0]}}+\frac{\mu w_{1}}{1+\tilde{p} \tilde{q}+\widetilde{w}_{0}}-\left(1+\frac{\mu w_{0}}{1+\tilde{p} \tilde{q}+\widetilde{w}_{0}}\right) \frac{v_{[0,1]}}{v_{[0,0]}}\right)\left(\mathbf{v}+\left(\tilde{p} \tilde{q}+\widetilde{w}_{0}\right) \mathbf{u}\right),
\end{aligned}
$$

где

$$
f^{2}=\frac{\mu^{2}\left(v_{[0,0]} u_{[0,0]}-w_{0}^{2}\right)}{\left(1+\tilde{p} \tilde{q}+\widetilde{w}_{0}\right)^{2}}+\frac{1-\tilde{p} \tilde{q}-\widetilde{w}_{0}}{1+\tilde{p} \tilde{q}+\widetilde{w}_{0}}-\frac{2\left(1+\mu w_{0}\right)}{1+\tilde{p} \tilde{q}+\widetilde{w}_{0}},
$$

$a \eta^{2}=v_{[0,0]}\left(1+a v_{[1,1]}\right)-a v_{[0,1]}^{2}, \tilde{p}^{2}=\tilde{u}_{[0,0]}+1, \tilde{q}^{2}=\tilde{v}_{[0,0]}+1$. Наконец, для анизотропного уравнения (17) мы построили следуюшее преобразование Беклунда:

$$
\mathbf{u}_{x}-\mathbf{v}_{x}=2\left[\widetilde{w}_{1}-\frac{h+g w_{0}+1}{g v_{[0,0]}} \tilde{v}_{[0,1]}-f\right] \mathbf{u}+e \mathbf{v},
$$

где

$$
\begin{gathered}
f=\frac{\eta+v_{[0,1]}}{4 v_{[0,0]}}\left(\frac{g v_{[0,0]}}{h+g w_{0}+1}\left(1+2 \tilde{u}_{[0,0]}\right)-\frac{h+g w_{0}+1}{g v_{[0,0]}}\left(1+2 \tilde{v}_{[0,0]}\right)\right)+ \\
+\frac{e}{4}\left(\frac{g v_{[0,0]}}{h+g w_{0}+1}\left(1+2 \tilde{u}_{[0,0]}\right)+\frac{h+g w_{0}+1}{g v_{[0,0]}}\left(1+2 \tilde{v}_{[0,0]}\right)-4 \widetilde{w}_{0}\right), \\
e=g w_{1}-v_{[0,1]} \frac{1+g w_{0}}{v_{[0,0]}}+\frac{h \eta}{v_{[0,0]}}, \\
h^{2}=\left(g w_{0}+1\right)^{2}-u_{[0,0]} v_{[0,0]} g^{2}, \\
-a \eta^{2}=v_{[0,0]}\left(1+a v_{[1,1]}\right)-a v_{[0,1]}^{2},
\end{gathered}
$$

а функция $g$ есть корень уравнения

$$
\begin{aligned}
\left(g w_{0}\right. & +1)^{2}-g^{2} u_{[0,0]} v_{[0,0]}= \\
= & \left(\frac{u_{[0,0]}+v_{[0,0]}+2\left(\mu u_{[0,0]} v_{[0,0]}+\tilde{v}_{[0,0]} u_{[0,0]}+\tilde{u}_{[0,0]} v_{[0,0]}\right)}{u_{[0,0]}-v_{[0,0]}+2\left(\tilde{v}_{[0,0]} u_{[0,0]}-\tilde{u}_{[0,0]} v_{[0,0]}\right)}-\right. \\
& \left.\quad-\frac{2 u_{[0,0]} v_{[0,0]}\left(1+2 \widetilde{w}_{0}\right)-v_{[0,0]} w_{0}\left(1+2 \tilde{u}_{[0,0]}\right)-u_{[0,0]} w_{0}\left(1+2 \tilde{v}_{[0,0]}\right)}{u_{[0,0]}-v_{[0,0]}+2\left(\tilde{v}_{[0,0]} u_{[0,0]}-\tilde{u}_{[0,0]} v_{[0,0]}\right)} g\right)^{2} .
\end{aligned}
$$

\section{4. ЗАКЛЮЧИТЕЛЬНЫЕ ЗАМЕЧАНИЯ}

Как отмечалось, уравнения (4) и (5) уже встречались в литературе, и их интегрируемость не вызывает сомнений. Существование связей между ними и системами (8), (9) явно свидетельствует об интегрируемости последних.

С помощью уравнения (20) можно легко построить односолитонное решение (7). Так как $\mathbf{u}=0$ - тривиальное решение уравнения (7), положим $\mathbf{v}=0$ в уравнении (20); тогда получим уравнение

$$
\mathbf{u}_{x}=-\mu \sqrt{a} \sqrt{a-u_{[0,0]}} \mathbf{u}
$$


и его следствия

$$
u_{[0,1]}=-\mu \sqrt{a} \sqrt{a-u_{[0,0]}} u_{[0,0]}, \quad u_{[1,1]}=\mu^{2} a\left(a-u_{[0,0]}\right) u_{[0,0]}
$$

Подставив выражение (23) в уравнение (7) и используя (22), мы получаем

$$
\mathbf{u}_{t}=-\mu^{3} a^{2} \sqrt{a} \sqrt{a-u_{[0,0]}} \mathbf{u}
$$

это означает, что $\mathbf{u}=\mathbf{u}\left(x+\mu^{2} a^{2} t\right)$. Из первого из уравнений (23) находим

$$
u_{[0,0]}=\frac{a}{\operatorname{ch}^{2}\left(\mu a\left(x+\mu^{2} a^{2} t\right)\right)}
$$

и окончательно имеем

$$
\mathbf{u}=\frac{\sqrt{a} \mathbf{C}}{\operatorname{ch}\left(\mu a\left(x+\mu^{2} a^{2} t\right)\right)}, \quad(\mathbf{C}, \mathbf{C})=1,
$$

где $\mathbf{C}$ - постоянный вектор.

Уравнение (15) есть аналог уравнения (2), интегрируемого на $n$-мерной сфере. Перейдем в уравнении (15) к координатам

$$
\mathbf{v}=\frac{\mathbf{u}}{u_{[0,0]}}, \quad r=\sqrt{u_{[0,0]}}
$$

тогда оно равносильно системе из одного векторного и одного скалярного уравнения:

$$
\begin{aligned}
\mathbf{v}_{t}= & \mathbf{v}_{x x x}+3 \frac{r_{x}}{r} \mathbf{v}_{x x}+3 \frac{r_{x x}}{r} \mathbf{v}_{x}+ \\
& +\frac{3}{2}\left(\frac{r^{2} v_{[1,1]}}{a}+\frac{r_{x}^{2}}{a-r^{2}}+r^{2} \tilde{v}_{[0,0]}\right) \mathbf{v}_{x}+3\left(v_{[1,2]}+\frac{r_{x} v_{[1,1]}}{r}\right) \mathbf{v} \\
r_{t}= & r_{x x x}+\frac{3 r r_{x}}{a-r^{2}} r_{x x}+ \\
& +\frac{3}{2}\left(\frac{\left(a+r^{2}\right) r_{x}^{2}}{\left(a-r^{2}\right)^{2}}-\frac{\left(2 a-3 r^{2}\right) v_{[1,1]}}{a}+r^{2} \tilde{v}_{[0,0]}\right) r_{x}-\frac{3\left(a-r^{2}\right) v_{[1,2]}}{a} r
\end{aligned}
$$

Осушествим в последней системе предельный переход при $r(x, t) \rightarrow \sqrt{a}$. Для этого положим сначала $r(x, t)=r \equiv$ const (например, в работе [4] $r=1$ ); тогда все производные скалярной функции $r(x, t)$ будут равны нулю. Затем устремим $r$ к $\sqrt{a}$ и в итоге получим одно векторное уравнение

$$
\mathbf{v}_{t}=\left(\mathbf{v}_{x x}+\frac{3}{2} v_{[1,1]} \mathbf{v}\right)_{x}+\frac{3}{2} \tilde{v}_{[0,0]} \mathbf{v}_{x}, \quad \mathbf{v}^{2}=1
$$


которое совпадает с уравнением (2). Константа $а$ включена в анизотропную метрику. Согласно перечисленным условиям преобразование Беклунда для уравнения (15) перейдет в преобразование Беклунда для уравнения (25):

$\mathbf{u}_{x}+\mathbf{v}_{x}=\frac{w_{1}}{1+w_{0}}(\mathbf{u}+\mathbf{v})+\frac{f}{1+w_{0}}\left(\mathbf{v}-w_{0} \mathbf{u}\right), \quad f^{2}=\mu\left(1+w_{0}\right)-\left(\tilde{u}_{[0,0]}+\tilde{v}_{[0,0]}+2 \widetilde{w}_{0}\right)$

В уравнении (16) также можно осуществить редукцию (24), что дает

$$
\begin{aligned}
\mathbf{v}_{t} & =\mathbf{v}_{x x x}+3 \frac{r_{x}}{r} \mathbf{v}_{x x}+3\left(\frac{r_{x x}}{r}+\frac{r^{2}}{a} v_{[1,1]}+\frac{r_{x}^{2}}{a-r^{2}}\right) \mathbf{v}_{x}+3\left(v_{[1,2]}+\frac{r_{x}}{r} v_{[1,1]}\right) \mathbf{v} \\
r_{t} & =r_{x x x}+\frac{3 r r_{x}}{a-r^{2}} r_{x x}-3\left(\frac{\left(a-r^{2}\right) r}{a} v_{[1,2]}+\frac{\left(a-2 r^{2}\right) r_{x}}{a} v_{[1,1]}-\frac{a r_{x}^{3}}{\left(a-r^{2}\right)^{2}}\right)
\end{aligned}
$$

Как и в предыдушей системе, перейдя к пределу $r(x, t) \rightarrow \sqrt{a}$, приходим к одному векторному уравнению

$$
\mathbf{v}_{t}=\mathbf{v}_{x x x}+3 v_{[1,1]} \mathbf{v}_{x}+3 v_{[1,2]} \mathbf{v}, \quad \mathbf{v}^{2}=1
$$

Уравнение (26) встречалось в работе [4], преобразование (20) переходит в преобразование Беклунда для (26) (ср. с [4])

$$
\mathbf{u}_{x}+\mathbf{v}_{x}=\frac{w_{1}}{1+w_{0}}(\mathbf{u}+\mathbf{v})+\mu\left(\mathbf{v}-w_{0} \mathbf{u}\right)
$$

Отметим также, что в анизотропных уравнениях (15)-(17) и соответственно в преобразованиях Беклунда для них скалярное произведение $\langle\cdot, \cdot\rangle$ можно брать как равным произведению $(\cdot, \cdot)$, так и равным нулю, причем для функции $h$ из уравнения $(17)$ эти две возможности равносильны.

Благодарности. Автор благодарит проф. А.Г. Мешкова за постановку задачи и полезные обсуждения, а также признателен рецензенту за критические замечания. Все вычисления проведены на базе центра компьютерной алгебры MuPad - университет Падербонр (Германия). Финансирование осушествлялось фондом Ханнса Зайделя. Работа частично поддержана также грантом РФФИ № 02-01-00431.

\section{Список литературы}

[1] С. И. Свинолупов, В. В. Соколов. ТМФ. 1994. Т. 100. № 2. С. 214-218.

[2] И. З. Голубчик, В. В. Соколов. ТМФ. 2000. Т. 124. № 1. С. 62-71.

[3] V. V. Sokolov, T. Wolf. J. Phys. A. 2001. V. 34. P. 11139-11148.

[4] A. G. Meshkov, V. V. Sokolov. Commun. Math. Phys. 2002. V. 232. № 2. P. 1-18.

[5] А. Г. Мешков, В. В. Соколов. ТМФ. 2004. Т. 139. № 2. С. 192-208.

[6] А. Г. Мешков. Software and help files for Maple. http://www.orel.ru/meshkov.

Поступила в редакцию 6.IV.2004 г., после доработки 5.V.2004 г. 